\title{
Influencia de la sensibilidad a la ansiedad en una intervención psicológica para dejar de fumar
}

\author{
Carmela Martínez-Vispo ${ }^{1}$, Elena Fernández del Río ${ }^{2}$, Ana López-Durán ${ }^{1}$ y Elisardo Becoña ${ }^{1}$ \\ ${ }^{1}$ Universidad de Santiago de Compostela, Santiago de Compostela, España \\ ${ }^{2}$ Universidad de Zaragoza, Zaragoza, España
}

\begin{abstract}
Resumen: Actualmente, fumar es uno de los principales factores de riesgo para la calidad de vida y la salud física y psicológica de las personas. Por otro lado, la sensibilidad a la ansiedad (SA) está implicada en la aparición y mantenimiento de la conducta de fumar, así como en el proceso de dejar de fumar y en el de recaída. El objetivo de este trabajo fue evaluar si la SA se relaciona con lograr la abstinencia al final de una intervención psicológica para dejar de fumar y con distintas variables del consumo de tabaco. Los resultados obtenidos mostraron que tras la intervención, los participantes que no consiguieron dejar de fumar tenían mayores puntuaciones en SA y mayor dependencia de la nicotina. Por tanto, la SA influye en el proceso de dejar de fumar, lo cual tiene implicaciones clínicas relevantes para el proceso de intervención y sus resultados.
\end{abstract}

Palabras clave: Fumar; intervención psicológica; sensibilidad a la ansiedad.

\section{Influence of anxiety sensitivity in a psychological smoking cessation intervention}

\begin{abstract}
Currently, smoking is a major risk factor for quality of life and physical and psychological health. On the other hand, anxiety sensitivity (AS) is involved not only in the development and maintenance of smoking behaviour, but also in smoking cessation and relapse. The aim of this study is to assess what correlation AS has to achieving abstinence at the end of a psychological intervention for smoking cessation, and to other smoking-related variables. After the intervention, participants who failed to quit smoking were those with higher AS scores and higher nicotine dependence. Thus, AS affects quitting smoking, which has important clinical implications for the intervention process and its results.
\end{abstract}

Keywords: Smoking; psychological intervention; anxiety sensitivity.

\section{Introducción}

Fumar constituye la primera causa evitable de enfermedad y muerte en el mundo (Organización Mundial de la Salud, 2013), situándose la mortalidad atribuible al consumo de tabaco en torno a las 50.000 personas al año en España (Banegas et al., 2011). Pero además, el tabaco constituye uno de los problemas de salud evitables más

Recibido: 5 febrero 2016; aceptado: 12 febrero 2016.

Correspondencia: Carmela Martínez Vispo, Facultad de Psicología, Universidad de Santiago de Compostela, Campus Vida. 15782 Santiago de Compostela.

Correo-e: carmela.martinez@usc.es

Agradecimientos: Deseamos agradecer al Dr. Bonifacio Sandín su contribución, sugerencias y revisión de la presente investigación. importante en los países desarrollados, atribuyéndose directamente al consumo de tabaco el 30\% de las muertes por cáncer, destacando el cáncer de la cavidad oral y faríngea, cáncer de laringe, cáncer de tráquea, bronquios y pulmón, cáncer de esófago, cáncer de estómago, cáncer de páncreas, cáncer de vejiga, cáncer de riñón y cáncer de cuello de útero. Del mismo modo, el tabaco es responsable de al menos el $30 \%$ de las muertes por enfermedad cardiovascular, y está relacionado con la incidencia y gravedad de las enfermedades respiratorias o gastrointestinales, entre otras (U.S.D.H.H.S., 2014).

En España, la prevalencia del consumo de tabaco todavía es una de las más elevadas entre los países europeos (OECD, 2014). Aunque los datos más recientes de la Encuesta Nacional de Salud, ENSE 2011-2012 indi- 
can que hemos alcanzado la cifra más baja de consumo de tabaco de los últimos 25 años, pasando de un $26.4 \%$ en 2006 a un 24\% en 2013 (Instituto Nacional de Estadística, 2013), todavía fuma un importante porcentaje de la población.

Sabemos, además, que el consumo de tabaco está asociado no sólo con un peor estado de salud física, sino que también influye en la salud psicológica. Distintos estudios han encontrado una relación entre fumar y diferentes trastornos mentales, entre los que destacan los trastornos del estado de ánimo (Callaghan et al., 2014), los trastornos de ansiedad (Moylan, Jacka, Pasco y Berk, 2012) y la esquizofrenia (Aubin, Rollema, Svensson y Winterer, 2012).

La SA puede definirse como el miedo a las sensaciones interoceptivas indicativas de activación o de otros síntomas de ansiedad, que surge de la creencia de que éstas señalan peligro $\mathrm{u}$ otras consecuencias temidas (McNally, 2002; Reiss y McNally, 1985).

Diferentes estudios han encontrado una relación entre el consumo de tabaco y la SA, considerándose como una variable de vulnerabilidad transdiagnóstica que podría estar detrás de la relación entre fumar y diferentes trastornos psicopatológicos (Leventhal y Zvolensky, 2015). Así, se ha encontrado que las personas con elevada SA es más probable que fumen, debido a la creencia de que fumar supone una herramienta de afrontamiento que les ayuda a regular estados emocionales negativos y aversivos (Brown, Kahler, Zvolensky, Lejuez y Ramsey, 2001; Guillot, Pang y Leventhal, 2014) y encuentran el proceso de dejar de fumar como más difícil y desafiante (Johnson, Farris, Schmidt, Smits y Zvolensky 2013), probablemente debido a las expectativas de que dejar de fumar incrementa el afecto negativo, el malestar y otros síntomas desagradables (Abrams, Zvolensky, Dorman, González y Mayer, 2011, Guillot et al., 2014). Por ejemplo, Zvolensky, Farris, Schmidt y Smits (2014) realizaron un estudio en una muestra de 466 fumadores que demandan tratamiento para dejar de fumar, encontrando que la SA se relacionó indirectamente con mayores barreras percibidas para dejar de fumar y con un mayor número de intentos previos de abandono.

También se ha encontrado que los fumadores con elevada SA experimentan síntomas del síndrome de abstinencia de la nicotina más intensos (Langdon et al., 2013) y una mayor probabilidad de no conseguir dejar de fumar tras un intento de abandono, así como con una mayor probabilidad de recaer y fumar en las semanas siguientes. Por ejemplo, Zvolensky, Stewart, Vujanovic, Gavric y Steeves, (2009), en un estudio en el que los participantes reciben consejo para dejar de fumar y Terapia Sustitutiva de la Nicotina (TSN), encontraron que, antes del abandono, la SA se asoció significativamente con un aumento del riesgo de caídas tempranas entre los días 1,7 y 14 después de la fecha de abandono, aunque no con la recaída completa (es decir, fumar durante 7 días consecutivos).

En la actualidad, sabemos que tanto las intervenciones conductuales como los tratamientos farmacológicos, combinados o por sí solos, son eficaces para dejar de fumar entre la población general adulta (Patnode et al., 2015). Sin embargo, en nuestro contexto hemos observado que los porcentajes de abstinencia en personas que acuden a una intervención psicológica para dejar de fumar se han reducido en los últimos años (Becoña, Lopez-Durán, Fernández del Río y Martínez, 2014). Esta reducción es probable que esté relacionada con un incremento de la comorbilidad entre el consumo de tabaco y otros trastornos psicopatológicos como los afectivos y los de ansiedad. Diferentes modelos han propuesto que algunos constructos psicológicos como la anhedonia, la tolerancia al malestar o la SA podrían tener un papel en dicha relación (Leventhal y Zvolensky, 2015).

En función de lo expuesto, el objetivo principal del presente estudio es evaluar las variables que influyen en el consumo de tabaco de los participantes en una intervención psicológica para dejar de fumar en relación con la variable SA, así como la influencia de esta variable en los resultados de abstinencia al final de la intervención.

\section{Método}

\section{Participantes}

La muestra del presente estudio estuvo formada por fumadores que demandaron ayuda para dejar de fumar entre enero y octubre de 2015 en la Unidad de Tabaquismo y Trastornos Adictivos de la Facultad de Psicología de la Universidad de Santiago de Compostela. Los criterios de inclusión en el estudio fueron: (1) tener 18 o más años, (2) desear participar voluntariamente en el programa para dejar de fumar, 3) presentar un consumo mínimo de 10 cigarrillos diarios antes del inicio de la intervención, y (4) cumplimentar debidamente todos los cuestionarios de la evaluación preintervención. Por otra parte, eran excluidas del estudio aquellas personas: (1) con diagnóstico de trastorno mental grave (trastorno bipolar y/o trastorno psicótico), (2) con dependencia concurrente de otras sustancias (p.ej., cannabis, cocaína, heroína, etc.), (3) que fumasen tabaco de liar, puros o puritos, (4) que hubieran participado en el programa para dejar de fumar o en otra intervención psicológica 
eficaz para dejar de fumar durante el año previo. (5) que hubieran recibido otro tipo de intervención efectiva para dejar de fumar en el último año (chicles o parches de nicotina, bupropión, vareniclina), (6) que padeciesen una patología de alto riesgo vital que precisaría una intervención inmediata en formato individual (ej. infarto de miocardio reciente, neumotórax, etc.), o (7) que no acudiese a la primera sesión de intervención en grupo. Siguiendo los criterios de inclusión y exclusión, de las 183 personas que demandaron información en la Unidad de Tabaquismo y Trastornos Adictivos, 121 completaron la evaluación, $\mathrm{y}$, finalmente, 92 pasaron a formar parte del estudio. La muestra final estaba constituida por 35 varones y 57 mujeres de entre 19 y 69 años (Media de edad $=44.50 ; D T=11.90)$.

\section{Instrumentos de evaluación}

La evaluación consistió en la administración de los siguientes cuestionarios:

Cuestionario sobre el hábito de fumar (Becoña, 1994), formado por 59 preguntas que analizan variables sociodemográficas (edad, sexo, estado civil, estudios, profesión, clase social e ingresos económicos) y de consumo de tabaco $\left(\mathrm{n}^{\mathrm{o}}\right.$ de cigarrillos al día, consumo de otros productos del tabaco como puros, puritos y pipas, edad a la que empezó a fumar, marca de cigarrillos, número de inhalaciones, cantidad del cigarrillo que fuma, intentos previos de abandono o reducción de cigarrillos, etc.).

Test de Fargerström de Dependencia de la Nicotina (Fagerström Test for Nicotine Dependence, FTND; Heatherton, Kozlowski, Frecker y Fagerström, 1991; adaptación al castellano de Becoña, 1994): formado por 6 ítems de dos a cuatro alternativas de respuesta con un punto de corte para la dependencia de la nicotina de 6 (Fagerström et al., 1996). En España la fiabilidad de este cuestionario se encuentra en .60 (Becoña y Lorenzo, 2004) y es el más utilizado para evaluar la dependencia de la nicotina. En el presente estudio se obtuvo un alfa de Cronbach de .64 .

Índice de SA-3 (Anxiety Sensitivity Index-3; Taylor et al. 2007, Sandín, Valiente, Chorot y Santed, 2007). Es una escala de autoinforme que consta de 18 ítems que evalúan las tres dimensiones de la SA: física (miedo a que los síntomas físicos de ansiedad sean dañinos), cognitiva (miedo a que los síntomas cognitivos de la ansiedad indiquen anormalidad mental), y social (miedo a que otras personas puedan notar los síntomas de ansiedad). Los índices de fiabilidad de la adaptación española van en la línea del estudio original, obteniendo coeficientes alfa excelentes tanto para la escala total como para las tres subescalas (coeficientes a de .84 a .91). En esta muestra el coeficiente alfa obtenido fue de .89. Además, la fiabilidad test-retest es excelente, con correlaciones que van de .83 a .85 .

Autorregistros: se trata de unas hojas que se les proporcionan a los participantes para que conozcan en profundidad su conducta de fumar. Los participantes deben registrar diariamente el número de cigarrillos, la hora en la que fuma el cigarrillo, el placer que le provoca (de 0 a 10 , siendo 0 el mínimo placer y 10 el máximo) y la situación concreta en la que se encuentra.

Evaluación del monóxido de carbono (CO) en aire espirado: se trata de una medida fácil de usar, no invasiva y que proporciona un feedback inmediato. Se ha uti-

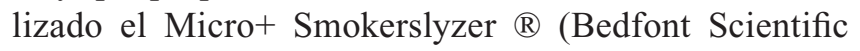
Instruments Ltd, Sittingbourne, Kent, Reino Unido), que es un aparato que proporciona una lectura digital de las partículas por millón (ppm) de $\mathrm{CO}$ en el aire espirado. En el presente estudio utilizaremos un punto de corte de 10 ppm o más para fumador, ya que es el más utilizado (West, Hajek, Stead y Stapleton, 2005).

Cuestionario de evaluación de final de tratamiento (Becoña y Míguez, 1995) en el que se evaluaron aspectos como: consumo actual de cigarrillos, fecha para dejar de fumar (en caso de no haber dejado de fumar), apoyo recibido por parte de las personas de su entorno para dejar de fumar puntuado en una escala de 0 a 5 (desde "ningún apoyo" hasta "total apoyo"), mejorías físicas y psicológicas experimentadas y escala de síntomas de abstinencia del tabaco de Hughes y Hatsukami (1986).

\section{Procedimiento}

El presente estudio se llevó a cabo entre personas fumadoras que demandaron ayuda de manera voluntaria para dejar de fumar en la Unidad de Tabaquismo y Trastornos Adictivos de la Facultad de Psicología de la Universidad de Santiago de Compostela entre enero y octubre de 2015. Los participantes fueron reclutados a través de diversas comunicaciones a la prensa, carteles situados en distintos puntos de la ciudad y de los centros de salud, a través de otros fumadores que habían acudido previamente a la misma, o debido a la remisión por parte de su médico de atención primaria u otros servicios especializados del sistema sanitario.

Una vez que los participantes solicitaban información, uno de los terapeutas les explicaba en qué consistía la intervención, y, si la persona se mostraba interesada, se concertaba una cita para la realización de la evaluación, que tenía una duración aproximada de dos sesiones de una hora y media cada una. Antes de realizar la eva- 
luación, se explicaba y firmaba el consentimiento informado.

Se aplicó la intervención psicológica cognitivo-conductual Programa para Dejar de Fumar (Becoña, 2007), a lo largo de 8 sesiones, una por semana. Esta intervención consta de los siguientes elementos: contrato terapéutico, autorregistros y representación gráfica del consumo de cigarrillos, información general sobre el tabaco y los fumadores, desvanecimiento (reducción gradual de la ingesta de nicotina y alquitrán), control de estímulos, estrategias para no padecer el síndrome de abstinencia de la nicotina, feedback fisiológico del consumo de cigarrillos mediante medición de monóxido de carbono (CO) en aire espirado en cada sesión y estrategias de prevención de la recaída.

Esta intervención ha obtenido en distintos estudios porcentajes de abstinencia que varían de entre el 58\% y el $85 \%$ al final de la intervención (Piñeiro, Fernández del Río, López-Durán, Martínez y Becoña, 2013). En los últimos años, se ha detectado una disminución de la eficacia de esta intervención, obteniéndose en los últimos estudios porcentajes de abstinencia al final de la intervención en torno al 51\% (Becoña et al., 2014; Piñeiro y Becoña, 2013), debido probablemente a que las personas que continúan fumando y demandan ayuda psicológica para dejar de fumar tienen una mayor dependencia de la nicotina y una mayor psicopatología concurrente.

\section{Análisis de datos}

Los análisis estadísticos de este trabajo se llevaron a cabo con el paquete estadístico SPSS para Windows (versión 20.0). Se empleó la distribución de frecuencias para analizar la prevalencia de las características sociodemográficas, las características del consumo de tabaco y el porcentaje de abstinencia. Para analizar las diferencias entre hombres y mujeres respecto a variables relacionadas con el consumo de tabaco (número de cigarrillos antes de la intervención y dependencia de la nicotina evaluada con el FTND), se utilizó la prueba $t$ de Student. En el caso de las variables categóricas [dependencia de la nicotina según el FTND (dependiente/no dependiente) y abstinencia al final de la intervención (abstinente/no abstinente)], se realizaron tablas de contingencia y se aplicó el estadístico chi-cuadrado.

Finalmente, para conocer qué variables pre-intervención (sociodemográficas y SA) eran predictoras del consumo de cigarrillos al final de la misma, se realizó un análisis de regresión logística binaria multivariante por pasos hacia delante condicional, adoptando como variable criterio el estatus de fumador al final de la intervención, siendo 1 = fumador y $0=$ no fumador.

\section{Resultados}

Características sociodemográficas y de consumo de tabaco

La muestra total estuvo formada por 92 fumadores (38.0\% hombres y $62.0 \%$ mujeres) de entre 19 y 69 años, con una media de edad de 44.50 años $(D T=11.90)$. En la Tabla 1 se especifican las características sociodemográficas de la muestra.

Tabla 1. Características sociodemográficas de la muestra

\begin{tabular}{|c|c|c|}
\hline & $N$ & $\%$ \\
\hline \multicolumn{3}{|l|}{ Edad } \\
\hline 45 años o menos & 46 & 50.0 \\
\hline Más de 45 años & 46 & 50.0 \\
\hline \multicolumn{3}{|l|}{ Sexo } \\
\hline Hombre & 35 & 38.0 \\
\hline Mujer & 57 & 62.0 \\
\hline \multicolumn{3}{|l|}{ Estado civil } \\
\hline Soltero & 31 & 33.7 \\
\hline Casado o vive en pareja & 45 & 48.9 \\
\hline Separado o divorciado & 11 & 12.0 \\
\hline Viudo & 4 & 4.3 \\
\hline \multicolumn{3}{|l|}{ Número de hijos } \\
\hline Ninguno & 31 & 33.7 \\
\hline 1 o más & 61 & 66.3 \\
\hline \multicolumn{3}{|l|}{ Nivel de estudios } \\
\hline Básicos & 19 & 20.7 \\
\hline Medios & 28 & 30.4 \\
\hline Superiores & 45 & 48.9 \\
\hline \multicolumn{3}{|l|}{ Clase social } \\
\hline Baja / Media-Baja & 21 & 22.8 \\
\hline Media & 62 & 67.4 \\
\hline Alta / Media-Alta & 9 & 9.8 \\
\hline
\end{tabular}

Con respecto a las variables de consumo de tabaco, se trata de una muestra de participantes que fumaba una media de 19.21 cigarrillos diarios $(D T=7.99)$ de marcas con contenido alto en nicotina $(M=0.75 \mathrm{mg} ; D T=0.12)$. La media de cigarrillos que habían fumado como máximo en un día a lo largo del último año era de 28.11 $(D T=11.87)$ y en su vida de $32.19(D T=13.16)$. En relación a la forma de fumar, el $94.6 \%$ inhalaba el humo, el $25.0 \%$ reconocía dar muchas caladas al ciga- 
rrillo, el 57.6\% bastantes y el $17.4 \%$ decía dar pocas. Por otra parte, el $35.9 \%$ fumaba los cigarrillos hasta el filtro, el $53.3 \%$ fumaba casi todo el cigarrillo y el $10.8 \%$ sólo fumaba la mitad o menos del cigarrillo.

Con respecto a la dependencia de la nicotina, empleando el Test de Dependencia de la Nicotina de Fagerström (FTND), el $55.4 \%$ de los participantes no eran dependientes de la nicotina (puntuación menor de 6), mientras que el 44.6\% sí lo eran (puntuación de 6 o más). La puntuación media en el FTND fue de 4.80 (DT $=2.21$ ).

\section{Resultados de la intervención para dejar de fumar}

Con respecto a la abstinencia, se consideró a un participante abstinente al final de la intervención si no había fumado en las 24 horas anteriores a la última sesión y su nivel de $\mathrm{CO}$ era menor de $10 \mathrm{ppm}$. En caso de no conseguir que el participante accediese a la verificación de $\mathrm{CO}$ en aire espirado o que no se consiguiese contactar con él, se le consideraba fumador al mismo nivel (en número de cigarrillos y contenido de nicotina) que en la evaluación inicial. Por tanto, siguiendo dicho criterio, el $62.0 \%$ de los participantes consiguieron dejar de fumar al final de la intervención.

\section{Variables relacionadas con el consumo de tabaco en función del sexo, edad y estudios}

En cuanto a las diferencias entre hombres y mujeres en distintas variables relacionadas con el consumo de tabaco (Tabla 2), sólo se observaron diferencias significativas en el número de cigarrillos pre-intervención $(t=$ $2.315 ; p \leq .05)$, siendo los hombres los que más cigarrillos fumaban $(M=21.89 ; D T=10.01)$, en comparación con las mujeres $(M=17.56 ; D T=5.97)$. No se encontraron diferencias significativas en función del sexo en la dependencia de la nicotina evaluada con el FTND, ni en los porcentajes de abstinencia al final de la intervención.

Con respecto a la edad (hasta 45 años y más de 45) y al nivel de estudios (básicos, medios y superiores), no se observaron diferencias significativas entre los grupos en el número de cigarrillos preintervención, en la dependencia de la nicotina evaluada con el FTND ni en los porcentajes de abstinencia al final de la intervención.

\section{Relación entre dependencia de la nicotina y abstinencia al final de la intervención}

En relación al efecto de la dependencia de la nicotina sobre la abstinencia al final de la intervención, se obtuvieron diferencias significativas con el FTND $\left(\chi^{2}=7.651\right.$;
Tabla 2. Variables relacionadas con el consumo de tabaco en función del sexo.

\begin{tabular}{|c|c|c|c|c|c|}
\hline & \multicolumn{2}{|c|}{$\begin{array}{l}\text { Hombres } \\
(n=35)\end{array}$} & \multicolumn{2}{|c|}{$\begin{array}{l}\text { Mujeres } \\
(n=57)\end{array}$} & \multirow[b]{2}{*}{$t$} \\
\hline & Media & $D T$ & Media & $D T$ & \\
\hline $\begin{array}{l}\mathrm{N}^{0} \text { cigarrillos } \\
\text { preintervención }\end{array}$ & 21.89 & 10.01 & 17.56 & 5.97 & $2.315^{*}$ \\
\hline \multirow[t]{2}{*}{ FTND } & 4.80 & 2.34 & 4.80 & 2.14 & -0.015 \\
\hline & $n$ & $\%$ & $n$ & $\%$ & $\chi^{2}$ \\
\hline \multicolumn{6}{|l|}{ FTND } \\
\hline $\begin{array}{l}\text { No dependiente } \\
(0-5)\end{array}$ & 21 & 60.0 & 30 & 52.6 & .477 \\
\hline $\begin{array}{l}\text { Dependiente } \\
\text { (6 o más) }\end{array}$ & 14 & 40.0 & 27 & 47.4 & \\
\hline \multicolumn{6}{|c|}{ Abstinencia fin intervención } \\
\hline Sí & 20 & 57.1 & 37 & 64.9 & .555 \\
\hline No & 15 & 42.9 & 20 & 35.1 & \\
\hline
\end{tabular}

Nota. FTND = Test de Fargerström de Dependencia de la Nicotina. $* p \leq .05$

$p \leq .01$ ) (Tabla 3), siendo los fumadores dependientes de la nicotina los que obtenían un menor porcentaje de abstinencia (33.3\%) respecto a los no dependientes (66.7\%). Así mismo, se encontraron diferencias significativas en las medias de la puntuación en el FTND, mostrando una mayor dependencia de la nicotina los participantes que seguían fumando al final de la intervención que los que estaban abstinentes.

Tabla 3. Porcentaje de abstinentes al final de la intervención en función de la dependencia de la nicotina evaluada con el FTND.

\begin{tabular}{lccccc}
\hline \multirow{2}{*}{ FTND } & \multicolumn{2}{c}{$\begin{array}{c}\text { Fuman } \\
(n=35)\end{array}$} & \multicolumn{2}{c}{$\begin{array}{c}\text { No Fuman } \\
(n=57)\end{array}$} & \\
\cline { 2 - 5 } & $n$ & $\%$ & $n$ & $\%$ & $\chi^{2}$ \\
\hline $\begin{array}{l}\text { Dependientes } \\
(p \geq 6)\end{array}$ & 22 & 62.9 & 19 & 33.3 & $7.651^{* *}$ \\
$\begin{array}{l}\text { No dependientes } \\
(p<6)\end{array}$ & 13 & 37.1 & 38 & 66.7 & \\
& Media & $D T$ & Media & $D T$ & $t$ \\
& 5.82 & 1.96 & 4.17 & 2.13 & $3.721^{* * *}$ \\
\hline
\end{tabular}

Nota. FTND $=$ Test de Fargerström de Dependencia de la Nicotina. ${ }^{* *} p \leq .01 ; * * * p \leq .001$

\section{Resultados obtenidos con las escalas ASI-3}

Con respecto a las puntuaciones medias de la muestra total de fumadores en la escala ASI-3 (Tabla 4), los parti- 
cipantes obtuvieron en la escala total una media de 21.90 $(D T=13.22)$, mientras que las medias en las tres subescalas fueron: en la subescala física $8.66(D T=6.41)$, en la cognitiva $4.95(D T=5.00)$ y en la social $8.28(D T=5.01)$.

Por otro lado, cuando comparamos las medias de las escalas ASI-3 en función de la dependencia de la nicotina, se observaron diferencias significativas (Tabla 4), siendo los fumadores dependientes de la nicotina, los que mostraron puntuaciones medias significativamente más altas en las subescalas física $(t=1.951 ; p \leq .05) \mathrm{y}$ social $(t=-2.061 ; p \leq .05)$. Con respecto a las diferencias entre las medias de las escalas ASI-3 en función de la abstinencia al final de la intervención, se observaron diferencias significativas en la subescala física $(t=2.549$; $p \leq .01)$, siendo los participantes que lograban la abstinencia los que presentaban puntuaciones menores.

Tabla 4. Puntuaciones medias en la escala total y en las subescalas física, cognitiva y social en función de la dependencia y logro de la abstinencia al final de la intervención

\begin{tabular}{|c|c|c|c|c|c|c|c|c|c|c|c|c|}
\hline & \multicolumn{2}{|c|}{$\begin{array}{l}\text { Muestra total } \\
\qquad(n=92)\end{array}$} & \multicolumn{2}{|c|}{$\begin{array}{l}\text { FTND } \geq 6 \\
\quad(n=51)\end{array}$} & \multicolumn{2}{|c|}{$\begin{array}{l}\text { FTND }<6 \\
\quad(n=41)\end{array}$} & & \multicolumn{2}{|c|}{$\begin{array}{l}\text { Fuman } \\
(n=35)\end{array}$} & \multicolumn{2}{|c|}{$\begin{array}{l}\text { No fuman } \\
(n=57)\end{array}$} & \multirow[b]{2}{*}{$t$} \\
\hline & Media & $D T$ & Media & $D T$ & Media & $D T$ & & Media & $D T$ & Media & $D T$ & \\
\hline ASI-3 Física & 8.663 & 6.419 & 10.09 & 6.28 & 7.50 & 6.35 & $-1.951^{*}$ & 10.94 & 7.47 & 7.26 & 5.27 & $2.549 * *$ \\
\hline ASI-3 Cognitiva & 4.956 & 5.007 & 5.00 & 4.81 & 4.92 & 5.20 & -0.074 & 5.51 & 5.81 & 4.61 & 4.45 & .836 \\
\hline ASI-3 Social & 8.282 & 5.0149 & 9.46 & 5.12 & 7.33 & 4.76 & $-2.061 *$ & 8.25 & 5.29 & $\mathrm{v} 8.29$ & 4.88 & -.038 \\
\hline ASI-3 Total & 21.902 & 13.227 & 24.56 & 12.68 & 19.76 & 13.38 & -1.748 & 24.71 & 15.96 & 20.17 & 11.03 & 1.612 \\
\hline
\end{tabular}

Nota. FTND = Test de Fargerström de Dependencia de la Nicotina ( $<6=$ no dependencia; $\geq 6=$ dependencia). ASI-3 = Anxiety Sensitivity Index 3 .

$* p \leq .05 ; * * p \leq .01 ; * * * p \leq .001$

\section{Predicción del resultado de la intervención para dejar de fumar}

Se realizó un análisis de regresión logística binaria multivariante (hacia delante condicional), adoptando como variable criterio la abstinencia al final del intervención $(1=$ Sí, $0=$ No). Se incorporaron al análisis las siguientes variables predictoras: sexo (hombre/mujer), edad (45 años o menos/ más de 45 años), nivel de estudios (básicos/medios/superiores), número de cigarrillos preintervención, dependencia de la nicotina (de- pendiente/no dependiente) y SA (ASI-3: ansiedad total, ansiedad física, ansiedad cognitiva y ansiedad social).

Como puede verse en la Tabla 5, encontramos que la ausencia de dependencia de la nicotina $(O R=3.54)$, una mayor puntuación en la subescala de ansiedad social $(O R=1.12)$ y una menor puntuación en la subescala de ansiedad física $(O R=0.87)$, se asocian a una mayor probabilidad de dejar de fumar con la intervención, clasificándose correctamente al $76.1 \%$ de todos los participantes.

Tabla 5. Análisis de regresión logística de la abstinencia al finalizar la intervención.

\begin{tabular}{lccccc}
\hline & $B$ & $E T$ & Wald $(1)$ & Exp $(B)$ & I C (95\%) \\
\hline FTND $(\mathrm{p}<6)$ & $1.264^{* *}$ & 0.491 & 6.618 & 3.540 & $1.351-9.27$ \\
ASI-3 Física & $-0.130^{* *}$ & 0.046 & 8.154 & 0.878 & $0.802-0.960$ \\
ASI-3 Social & $0.120^{*}$ & 0.060 & 4.078 & 1.128 & $1.004-1.267$ \\
Constante & 0.005 & 0.592 & .000 & 1.005 & \\
\hline
\end{tabular}

Nota. FTND = Test de Fargerström de Dependencia de la Nicotina $(<6=$ no dependencia). ASI-3 = Anxiety Sensitivity Index 3.

$* p \leq .05 ; * p \leq .01$

\section{Discusión}

El objetivo principal de este trabajo fue evaluar la influencia de la SA en relación con diferentes características del consumo de tabaco, así como con el logro de la abstinencia al final de una intervención psicológica para dejar de fumar.

El porcentaje de abstinencia obtenido en este estudio al final de la intervención está en el rango de porcentajes de abstinencia (50-60\%) encontrado en otros estudios 
sobre intervenciones psicológicas para dejar de fumar (Becoña et al., 2014).

Con respecto a las puntuaciones medias de la muestra total de fumadores en la escala ASI-3, los participantes obtuvieron medias claramente superiores a los datos normativos de la versión española de la ASI-3, realizada en una muestra de 582 estudiantes universitarios (Sandín et al., 2007), la cual indica una media de $14.1(D T=9.6)$ para la ASI-3 total, de $4.5(D T=4.1)$ para la subescala física, $2.7(D T=3.5)$ para la subescala cognitiva y 6.9 $(D T=4.6)$ para la subescala social. Estos datos están en la línea de algunos estudios que han encontrado puntuaciones mayores en SA en fumadores en comparación con no fumadores (Abrams, et al., 2011; McLeish, Zvolensky, Yartz y Leyro, 2008).

Por otro lado, cuando se comparan las medias de la escala ASI-3 total y las de las subescalas en función de la dependencia de la nicotina, se observan diferencias significativas en la subescala física y social. Así, aquellos fumadores dependientes de la nicotina mostraron puntuaciones medias significativamente más altas en dichas escalas, resultados que siguen la línea de otros estudios como el de Brandt et al., (2015), realizado en una muestra de fumadores diarios que demandan intervención para dejar de fumar, y en el que los datos obtenidos muestran relación entre la dependencia de la nicotina y la SA.

Con respecto a las diferencias entre las medias de las escalas ASI-3 en función de lograr dejar de fumar, se observaron diferencias significativas en la subescala física. Así, aquellos fumadores que no consiguieron dejar de fumar al final de la intervención, mostraron puntuaciones medias significativamente más elevadas en la subescala física, en comparación con los que lograron dejar de fumar. Estos datos podrían explicarse en base a los resultados de estudios previos que señalan que los fumadores con elevada SA, tienen mayores expectativas de amenaza ante los síntomas interoceptivos de abstinencia (Farris, Langdon, DiBello y Zvolensky, 2014) y una tendencia a usar el consumo de tabaco como una estrategia para aliviar los síntomas aversivos del síndrome de abstinencia de la nicotina (ansiedad, irritabilidad, inquietud, craving, etc.), así como las emociones negativas asociadas (Zvolensky et al., 2014). Por tanto, los datos de nuestro estudio están en consonancia con la literatura previa que relaciona puntuaciones elevadas en SA con una menor probabilidad de dejar de fumar.

Por otro lado, aunque no era uno de los objetivos principales de este estudio, se consideró la relación entre la dependencia de la nicotina y la probabilidad de conseguir la abstinencia al final de la intervención, ya que en la literatura ésta resulta un factor reconocido por su in- fluencia en el mantenimiento de la conducta de fumar (Benowitz, 2010; Hagimoto, Nakamura, Morita, Masui y Oshima, 2009). Se encontraron diferencias estadísticamente significativas con respecto al efecto de la dependencia de la nicotina sobre la abstinencia al final de la intervención, siendo aquellos fumadores no dependientes de la nicotina los que obtuvieron un mayor porcentaje de abstinencia.

Finalmente, los datos obtenidos mediante el análisis de regresión logística binaria multivariante, indican que la ausencia de dependencia de la nicotina $(O R=3.54)$, una mayor puntuación en la subescala de ansiedad social $(O R=1.12)$ y una menor puntuación en la subescala de ansiedad física $(O R=0.83)$, predicen la abstinencia al final de la intervención, con un porcentaje de ajuste del modelo de regresión del 79.1\%. Estos resultados son acordes a los obtenidos en estudios previos en los que se ha encontrado que aquellos fumadores con una elevada SA, física experimentan mayores síntomas del síndrome de abstinencia de la nicotina (ansiedad, irritabilidad, inquietud, dificultades para dormir, etc.) lo cual dificulta el logro de la abstinencia y está implicado en el proceso de recaída temprana (Guillot, Zvolensky y Leventhal, 2015; Langdon et al., 2013). Parece que los fumadores con elevada sensibilidad, específicamente en la subescala física, son hipersensibles a las sensaciones interoceptivas relacionadas con los síntomas de abstinencia de la nicotina (Marshall, Johnson, Bergman, Gibson y Zvolensky, 2009). Un resultado que no esperábamos fue que las puntuaciones elevadas en SA social predijeran la abstinencia. Estudios previos han relacionado esta subescala con las expectativas de reforzamiento positivo y con la creencia de que fumar mejora el confort social (Buckner y Vinci, 2013; Guillot, Leventhal, Raines, Zvolensky y Schmidt, 2016), pero todavía no se conoce bien su papel en el proceso de dejar de fumar, por lo que debe seguir profundizándose en su estudio.

Las implicaciones de los datos obtenidos son relevantes a nivel clínico. Conocer como estas variables influyen en el proceso de abandono del tabaco, así como en el logro del mantenimiento de la abstinencia puede ayudar a mejorar las intervenciones para dejar de fumar, con la incorporación de componentes específicos dedicados al manejo de estas variables en aquellos fumadores con elevada SA. De hecho, algunos estudios han sugerido que la incorporación de elementos terapéuticos dirigidos específicamente a la SA en el contexto de una intervención psicológica para dejar de fumar, puede mejorar los resultados de abstinencia y adherencia a la intervención (Feldner, Zvolensky, Babson, Leen-Feldner y Schmidt, 2008; Zvolensky, Bogiaizian, Salazar, Farris y Bakhshaie, 2014). 
Podemos concluir, por tanto, que la SA influye en el proceso de dejar de fumar y en el logro de la abstinencia tras una intervención psicológica para dejar de fumar. Sin embargo, es necesario continuar estudiando esta relación en muestras de mayor tamaño y considerando otras variables que también podrían tener un papel relevante en el logro y el mantenimiento a largo plazo de la abstinencia, así como su influencia con respecto a la relación entre tabaco y los trastornos emocionales y de ansiedad.

Para terminar, este trabajo no está exento de limitaciones. En primer lugar, la muestra de fumadores empleada en este estudio estuvo formada por personas que demandaron ayuda psicológica para dejar de fumar, por lo que la muestra podría no ser representativa de toda la población de fumadores. Además, el tamaño de la muestra, aunque suficiente para los análisis realizados, es relativamente pequeño. Por otro lado, los datos se corresponden con el final de la intervención y no se realizaron seguimientos a largo plazo, lo cual podría ser de gran interés para el análisis del papel de la SA con respecto al proceso de mantenimiento de la abstinencia, así como en el proceso de recaída. Por último, no se analizaron otras variables que también pueden influir en el logro y mantenimiento de la abstinencia, como por ejemplo, el craving, la autoeficacia, la autoestima, el ajuste psicológico o la presencia de trastornos de personalidad, variables que ya han mostrado su relevancia en el tratamiento del tabaquismo y de otras adicciones (Martínez-González, Albein-Urios, Lozano-Rojas y Verdejo-García, 2015).

La investigación futura tiene como reto el continuar estudiando las variables implicadas en el logro de dejar de fumar, así como desarrollar protocolos de intervención psicológica que tengan en cuenta estas variables, evaluando su eficacia y explorando su relevancia para la prevención y abandono del tabaquismo. Así mismo, debemos seguir investigando la relación entre estas vulnerabilidades emocionales, los trastornos emocionales y de ansiedad y su coocurrencia con conductas relacionadas con la salud física y psicológica como es el consumo de tabaco.

\section{Referencias}

Abrams, K., Zvolensky, M. J., Dorman, L., González, A., \& Mayer, M. (2011). Development and validation of the Smoking Abstinence Expectancies Questionnaire. Nicotine \& Tobacco Research, 13, 1296-1304 doi: 10.1093/ntr/ntr184

Aubin, H. J., Rollema, H., Svensson, T. H., \& Winterer, G. (2012). Smoking, quitting, and psychiatric disease: A review. Neuroscience and Behavioral Reviews, 36, 271-284. doi:10.1016/j. neubiorev.2011.06.007

Banegas, J. R., Díez-Gañán, L., Bañuelos-Marco, B., González-Enríquez, J., Villar-Álvarez, F., Martín-Moreno, J. M., ...
Jiménez-Ruíz, C. (2011). Mortalidad atribuible al consumo de tabaco en España en 2006. Medicina Clínica, 136, 97-102.

Becoña, E. (2007). Programa para dejar de fumar. Vigo: Nova Galicia Edicións.

Becoña, E. (1994). Evaluación de la conducta de fumar. En J.L. Graña (ed.), Conductas adictivas. Teoría, evaluación y tratamiento (pp. 403-454). Madrid: Debate.

Becoña, E., López-Durán, A., Fernández del Río, E., \& Martínez, Ú. (2014). Changes in the profiles of smokers seeking cessation treatment and in its effectiveness in Galicia (Spain) 2001-10. BMC Public Health, 14, 613-622. doi:10.1186/1471-2458-14-613

Becoña, E., y Lorenzo, M. C. (2004). Evaluación de la conducta de fumar. Adicciones, 16 (Supl. 2), 201-226.

Becoña, E., y Míguez, M. C. (1995). El cuestionario de evaluación de la recaída/ abstinencia de los cigarrillos: primeros resultados. Revista Española de Drogodependencias, 20, 25-40.

Benowitz, N. L. (2010). Nicotine addiction. The New England JournalofMedicine, 362,2295-2303.doi:10.2466/pr0.1998.83.3f.1455

Brandt, C. P., Bakhshaie, J., Garey, L., Schmidt, N. B., Leventhal, A. M., \& Zvolensky, M. J. (2015). The moderating role of smoking amount per day on the relations between anxiety sensitivity, smoking dependence, and cognitive-affective aspects of smoking among treatment seeking smokers. Addictive Behaviors Reports, 1, 26-33. doi: 10.1016/j.abrep.2015.03.006

Brown, R. A., Kahler, C. W., Zvolensky, M. J., Lejuez, C. W., \& Ramsey, S. E. (2001). Anxiety sensitivity: Relationship to negative affect smoking and smoking cessation in smokers with past major depressive disorder. Addictive Behaviors, 26, 887899. doi:10.1016/S0306-4603(01)00241-6

Buckner, J. D., \& Vinci, C. (2013). Smoking and social anxiety: The roles of gender and smoking motives. Addictive Behaviors, 38, 2388-2391. doi: 10.1016/j.addbeh.2013.03.007

Callaghan, R. C., Veldhuizen, S., Jeysingh, T., Orlan, C., Graham, C., Kakouris, G.,...Gatley, J. (2014). Patterns of tobacco-related mortality among individuals diagnosed with schizophrenia, bipolar disorder, or depression. Journal of Psychiatric Research, 48, 102-110. doi: 10.1016/j.jpsychires.2013.09.014

Fagerström, K. O., Kunze, M., Schoberberger, R., Breslau, N., Hughes, J. R., Hurt, R. ... Zatonski, W. (1996). Nicotine dependence versus smoking prevalence: Comparison among countries and categories of smokers. Tobacco Control, 5, 5256. doi: 10.1136/tc.5.1.52.

Farris, S. G., Langdon, K. J., DiBello, A. M., \& Zvolensky, M. J. (2014). Why Do Anxiety Sensitive Smokers Perceive Quitting as Difficult? The Role of Expecting "Interoceptive Threat" During Acute Abstinence. Cognitive Therapy and Research, 39, 236-244. doi: 10.1007/s10608-014-9644-6

Feldner, M. T., Zvolensky, M. J., Babson, K., Leen-Feldner, E. W., $\&$ Schmidt, N. B. (2008). An integrated approach to panic prevention targeting the empirically supported risk factors of smoking and anxiety sensitivity: Theoretical basis and evidence from a pilot project evaluating feasibility and short-term efficacy. Journal of Anxiety Disorders, 22, 1227-1243. doi:10.1016/j.janxdis.2008.01.005.

Guillot, C. R., Leventhal, A. M., Raines, A. M., Zvolensky, M. J., y Schmidt, N. B. (2016). Anxiety sensitivity facets in relation to tobacco use, abstinence-related problems, and cognitions in treatment-seeking smokers. Addictive Behaviors, 56, 30-35. doi: 10.1016/j.addbeh.2016.01.005 
Guillot, C. R., Pang, R. D., \& Leventhal, A. M. (2014). Anxiety sensitivity and negative urgency: A pathway to negative reinforcement-related smoking expectancies. Journal of Addiction Medicine, 8, 189-194. doi: 10.1097/ADM.0000000000000017

Guillot, C. R., Zvolensky, M. J., \& Leventhal, A. M. (2015). Differential associations between components of anxiety sensitivity and smoking-related characteristics. Addictive Behaviors, 40, 39-44. doi: 10.1016/j.addbeh.2014.08.004

Hagimoto, A., Nakamura, M., Morita, T., Masui, S., \& Oshima, A. (2009). Smoking cessation patterns and predictors of quitting smoking among the Japanese general population: A 1-year follow-up study. Addiction, 105, 164-173.doi:10.1111/j.1360-0443.2009.02735.x

Heatherton, T. F., Kozlowski, L. T., Frecker, R. C., \& Fagerström, K. O. (1991). The Fagerström Test for Nicotine Dependence: A revision of the Fagerström Tolerance Questionnaire. British Journal of Addictions, 86, 1119-1127.

Hughes, J. R., \& y Hatsukami, D. K. (1986). Signs and symptoms of tobacco withdrawal. Archives of General Psychiatry, 43, 289-294. doi: 10.1001/archpsyc.1986.01800030107013

Instituto Nacional de Estadística (2013). Encuesta Nacional de Salud: ENSE 2011-2012. Recuperado de:

www.msc.es/estadEstudios/estadisticas/encuestaNacional/encuestaNac2011/NotaTecnica2011-12.pdf

Johnson, K. A., Farris, S. G., Schmidt, N. B., \& Zvolensky, M. J. (2012). Anxiety sensitivity and cognitive-based smoking processes: Testing the mediating role of emotion dysregulation among treatment-seeking daily smokers. Journal of Addictive Diseases, 31, 143-157. doi: 10.1080/10550887.2012.665695.

Langdon, K. J., Leventhal, A. M., Stewart, S., Rosenfield, D., Steeves, D., \& Zvolensky, M. J. (2013). Anhedonia and anxiety sensitivity: Prospective relationships to nicotine withdrawal symptoms during smoking cessation. Journal of Studies on Alcohol and Drugs, 74, 469-478. doi:10.1037/a0024133

Leventhal, A. M., \& Zvolensky, M. J. (2015). Anxiety, Depression, and Cigarette Smoking: A Transdiagnostic Vulnerability Framework to Understanding Emotion-Smoking Comorbidity. Psychological Bulletin, 141, 176-212. doi: 10.1037/bul0000003

Marshall, E. C., Johnson, K., Bergman, J., Gibson, L. E., \& Zvolensky, M. J. (2009). Anxiety sensitivity and panic reactivity to bodily sensations: Relation to quit-day (acute) nicotine withdrawal symptom severity among daily smokers making a self-guided quit attempt. Experimental and Clinical Psychopharmacology, 17, 356-364. doi: 10.1037/a0016883

Martínez-González, J. M., Albein-Urios, N., Lozano-Rojas, O., y Verdejo-García, A. (2015). Variables de interés clínico en el tratamiento cognitivo-conductual de la adicción a la cocaína: Especificidad de los trastornos de la personalidad. Revista de Psicopatología y Psicología Clínica, 20, 115-123. doi: 10.5944/rppc.vol.20.num.2.2015.15166

McLeish, A. C., Zvolensky, M. J., Yartz, A. R., \& Leyro, T. M. (2008). Anxiety sensitivity as a moderator of the association between smoking status and anxiety symptoms and bodily vigilance: Replication and extension in a young adult sample. Addictive Behaviors, 33, 315-327. doi: 10.1016/j.addbeh.2007.09.018

McNally, R. J. (2002). Anxiety sensitivity and panic disorder. Biological Psychiatry, 52, 938-946. doi:10.1016/S0006-3223(02)01475-0
Moylan, S., Jacka, F. N., Pasco, J. A., \& Berk, M. (2012). Cigarette smoking, nicotine dependence and anxiety disorders: a systematic review of population-based, epidemiological studies. BMC Medicine, 10, 123. doi:10.1186/1741-7015-10-123

OECD (2014). Health at a Glance: Europe 2014. OECD Publishing. Recuperado de: www.oecd.org/health/health-at-a-glance-europe-23056088.htm

Organización Mundial de la Salud (2013). Centro de prensa. Recuperado de: http://www.who.int/mediacentre/factsheets/fs339/es/

Patnode, C. D., Henderson, J. T., Thompson, J. H., Senger, C. A., Fortmann, S. P., \& Whitlock, E. P. (2015). Behavioral Counseling and Pharmacotherapy Interventions for Tobacco Cessation in Adults, Including Pregnant Women: A Review of Reviews for the US Preventive Services Task Force. Annals of Internal Medicine, 163, 608-621. doi:10.7326/M15-0171

Piñeiro, B., \& Becoña, E. (2013). Relapse situations according to Marlatt's taxonomy in smokers. Spanish Journal of Psychology, 16, 1-12. doi: 10.1017/sjp.2013.91

Piñeiro, B., Fernández del Río, E., López-Durán, A., Martínez, U., \& Becoña, E. (2013). The association between probable personality disorders and smoking cessation and maintenance. Addictive Behaviors, 38, 2369-2373. doi: 10.1016/j.addbeh.2013.03.017

Reiss, S., \& McNally, R. J. (1985). The expectancy model of fear. In S. Reiss y R. R. Bootzin (Eds.), Theoretical issues in behavior therapy (pp. 107-121). New York: Academic Press.

Sandín, B., Valiente, R. M., Chorot, P., y Santed, M. A. (2007). ASI-3: Nueva escala para la evaluación de la SA. Revista de Psicopatología y Psicología Clínica, 12, 91-104. Doi: 10.5944/rppc.vol.12.num.2.2007.4036

Taylor, S., Zvolensky, M. J., Cox, B. J., Deacon, B., Heimberg, R. G., Ledley, D. R., ... Cardenas, S. J. (2007). Robust dimensions of anxiety sensitivity: development and initial validation of the Anxiety Sensitivity Index-3. Psychological Assessment, 19, 176-188. Doi: 10.1037/1040-3590.19.2.176

U.S.D.H.H.S. (2014). The health consequences of smoking-50 years of progress. A report of the Surgeon General. Rockville, MD: U. S. Department of Health and Human Services, Public Health Services, Office of the Surgeon General.

West, R., Hajek, P., Stead, L., \& Stapleton, J. (2005). Outcome criteria in smoking cessation trials: Proposal for a common standard. Addiction, 100, 299-303.doi:10.1111/j.1360-0443.2004.00995.x.

Zvolensky, M. J., Bogiaizian, D., Salazar, P. L., Farris, S. G., \& Bakhshaie, J. (2014). An anxiety sensitivity reduction smoking-cessation program for Spanish-speaking smokers (Argentina). Cognitive and Behavioral Practice, 21, 350-363. doi: 1077-7229/13/350-363\$1.00/0

Zvolensky, M. J., Farris, S. G., Schmidt, N. B., \& Smits, J. A. (2014). The role of smoking inflexibility/avoidance in the relation between anxiety sensitivity and tobacco use and beliefs among treatment-seeking smokers. Experimental and Clinical Psychopharmacology, 22, 229-237. doi: 10.1037/a0035306

Zvolensky, M. J., Stewart, S. H., Vujanovic, A. A., Gavric, D., \& Steeves, D. (2009). Anxiety sensitivity and anxiety and depressive symptoms in the prediction of early smoking lapse and relapse during smoking cessation treatment. Nicotine \& Tobacco Research, 11, 323-331. doi: 10.1093/ntr/ntn037 
